THE ANNALS OF "DUNĂREA DE JOS" UNIVERSITY OF GALATI

FASCICLE III, 2020, VOL. 43, NO. 1, ISSN 2344-4738, ISSN-L 1221-454X

ELECTROTECHNICS, ELECTRONICS, AUTOMATIC CONTROL, INFORMATICS

Article DOI: https://doi.org/10.35219/eeaci.2020.1.02

\title{
EVALUATION OF HARMONIC EFFECTS ON THE POWER SYSTEM DISTRIBUTION
}

\author{
Nicolae Badea ${ }^{1}$, Valentin Stefanescu ${ }^{2}$ \\ ${ }^{I}$ Dunarea de Jos University of Galati, Romania, ${ }^{2}$ SDEE Electrica, Romania
}

\begin{abstract}
The paper addresses the impact of harmonic effects on the distribution of energy in a medium voltage network $(20 \mathrm{kV})$. The objective of the paper is to highlight the effect of harmonic currents on transformer heating and transformer load capability when the load is nonlinear. The results of the measurements made during the $24 \mathrm{~h}$ period are analysed in terms of reducing the power of the transformer, increasing the temperature of the transformer oil and the powers circulation. The paper proposes two relations of calculation of the power losses of the transformer in the case of loads not included in the standardized limits of the power factor and the harmonic content. A simple and largely used way of splitting liabilities and costs of the issues relating to power quality, including reactive power, is to claim that voltage is the power supplier's responsibility, while current is the consumer's responsibility. Harmonic current is generally a distribution issue and less a transmission one. A large amount of (harmonic) losses shall occur at the nearest load transformer. Result of the analysis of the measured values and the calculations performed de-rating of the apparent power percentage is between $24-32 \%$, increasing the temperature of the transformer oil with $6 \%$ and the circulation of powers shown high values of the deforming power between $60-70 \mathrm{kVAD}$ at an average active power of $950 \mathrm{~kW}$. Finally, the paper proposed calculation relationships for energy loss assessment due to non-compliance of total harmonic current distortion $\left(\mathrm{THD}_{\mathrm{i}}\right)$ and power factor within the limits imposed by standards.
\end{abstract}

Keywords: Power system, Total harmonics distortion, power quality, energy loss.

\section{INTRODUCTION}

The presence of current and voltage harmonics in power systems is caused by power electronics. Due to their formidable advantages in terms of efficiency and controllability, electronic power converters are expected to grow significantly in the future and they can be found at all power and voltage levels. Harmonic currents are generated whenever a nonlinear load is connected to the distribution system. Harmonic currents, generated by non-linear loads, produce 'penalty losses' in every element of an electrical distribution system [1]. These harmonicrelated losses reduce system efficiency, cause apparatus overheating, and increase power and air conditioning costs. The major issues caused by harmonic currents include conductor overheating, especially that of neutral conductor (3rd order harmonics are homopolar components which close in the neutral conductor), overheating and vibration of induction motors and high loss in transformers. Harmonics present in the distribution systems causes 
additional temperature rise and hence loss of useful life of power cables. The paper [3] evaluated the effects of harmonics in distribution systems on the temperature rise and useful life of XLPE power cables. Harmonic distortion and associated economic losses mainly the potential economic losses due to equipment fail or malfunction that caused by high levels of harmonic distortions is quantified by a time varying coefficient[4], which is derived from daily load curve. The impact of different harmonic loads on distribution transformers was analyzed at laboratory level [5] with low rated power and low voltage showing that loss components affected by non-sinusoidal currents are: Joule loss, eddy current loss and the stray losses. The most notable are 5th and 7th current harmonics. Results of the harmonic loads about $50 \%$ of a transformer loss of life is caused by thermal stress which is produced by the nonlinear load [6]. Harmonic currents might damage power factor correction capacitors, if installed, and particular care should be taken in order to avoid resonance with the power distribution inductance. The industry has responded with two general solutions to the effects of harmonics on transformers: install a K-factor rated transformer or de-rated a standard transformer. The de-rating curve(figure1), taken from [1], shows that a transformer with $60 \%$ of its loads consisting of switched-mode power supplies, which is certainly possible in a commercial office building, should in fact be de-rated by $50 \%$

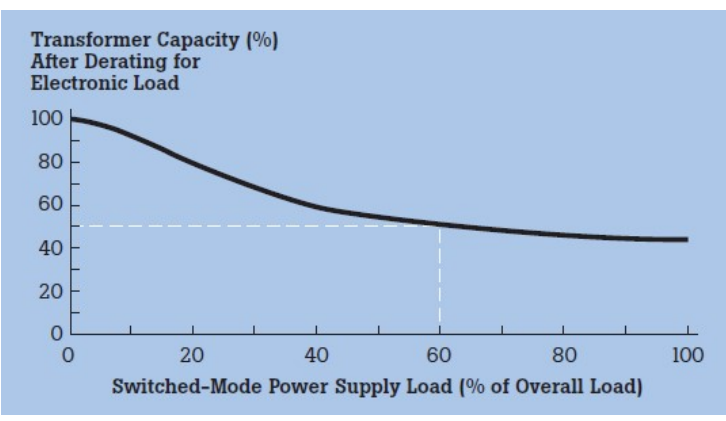

Fig.1. Transformer de-rating curve[1]

Title IEEE 519-1992 standard provides extensive recommendations for investigating, evaluating and measuring harmonics in the distribution system. Table 1 show the harmonic current distortion level and Table 2 shows the harmonic voltage distortion limits.

$\underline{\text { Table } 1 \text { The harmonic current distortion limits. }}$

\begin{tabular}{|c|c|c|c|c|c|c|}
\hline $\mathrm{I}_{\mathrm{SC}} / \mathrm{I}_{\mathrm{L}}$ & $\begin{array}{c}\mathrm{h}< \\
11\end{array}$ & $\begin{array}{c}11 \leq \mathrm{h} \\
<17\end{array}$ & $\begin{array}{c}17 \leq \mathrm{h} \\
<23\end{array}$ & $\begin{array}{c}23 \leq \mathrm{h}< \\
35\end{array}$ & $35 \leq \mathrm{h}$ & $\begin{array}{c}\mathrm{THD} \\
\mathrm{i}\end{array}$ \\
\hline$<20$ & 4.0 & 2.0 & 1.5 & 0.6 & 0.3 & 5.0 \\
\hline $20<50$ & 7.0 & 3.5 & 2.5 & 1.0 & 0.5 & 8.0 \\
\hline $50<100$ & 10 & 4.5 & 4.0 & 1.52 & 0.7 & 12.0 \\
\hline $100<10^{3}$ & 12 & 5.5 & 5.0 & 2.0 & 1.0 & 15.0 \\
\hline
\end{tabular}

Table 2 The harmonic voltage distortion limits.

\begin{tabular}{|c|c|c|}
\hline $\begin{array}{c}\text { Bus voltage at the } \\
\text { point of common } \\
\text { coupling }\end{array}$ & $\begin{array}{c}\text { Individual voltage } \\
\text { distortion }\end{array}$ & $\begin{array}{c}\text { Total harmonic } \\
\text { distortion } \text { THD }_{\mathrm{u}}\end{array}$ \\
\hline$\leq 69 \mathrm{kV}$ & 3.0 & 5.0 \\
\hline $69 \mathrm{kV}<161 \mathrm{kV}$ & 1.5 & 2.5 \\
\hline$>161 \mathrm{kV}$ & 1.0 & 1.5 \\
\hline
\end{tabular}

\section{LOSSES IN TRANSFORMERS CONFRONTED WITH HARMONICS}

Many transformers designed to operate at rated frequency have had their loads gradually replaced with non-linear loads, which inject harmonic currents. Such harmonic currents shall result in an increase of losses and, hence, in an abnormal temperature rise, which will shorten the expected lifetime. Transformer losses are caused by the magnetic loss in the core, which is proportional to frequency, and also by eddy current loss, which is directly proportional to the frequency square, to which the Joule loss in windings is added.

Transformer losses can be also generally classified into Load - No-load losses in accord with equation (1) [7].

$$
\text { (1) } \quad P_{T}=P_{\text {load }}+P_{\text {no-load }}
$$

The harmonic equivalent circuit of transformer is shown in Figure 2. Where, Rh (1), Rh (2), Xh (1), $\mathrm{Xh}$ (2) are winding equivalent resistance values and reactance values at order $\mathrm{h}$ respectively; $\mathrm{Rh}(\mathrm{m})$ and $\mathrm{Xh}(\mathrm{m})$ are magnetic resistance and reactance.

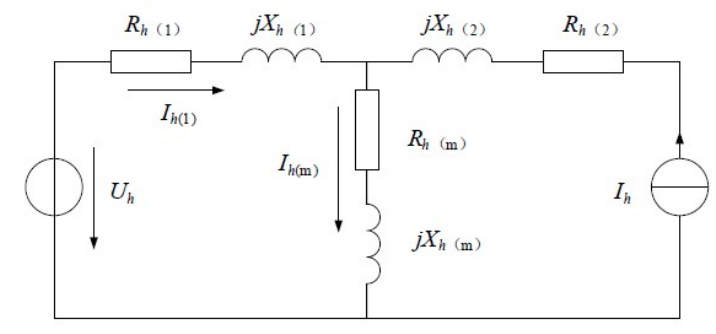

Fig.2 The harmonic equivalent circuit of transformer [8]

In three-phase systems, multiples of the $3^{\text {rd }}$ harmonic, $(h=3 n)$ generate a homopolar system, those of $h=3 n+1$ order generate a direct sequence system, and those of $\mathrm{h}=3 n-1$ order generate an inverse sequence system.

Electro technical curves contain only odd-order harmonics, therefore:

- $1^{\text {st }}, 7^{\text {th }}, 13^{\text {th }}, \ldots$ order harmonics generate direct sequence systems;

- $5^{\text {th }}, 11^{\text {th }}, 17^{\text {th }}, \ldots$ order harmonics generate inverse sequence systems;

- $3^{\text {rd }}, 9^{\text {th }}, 15^{\text {th }}, \ldots$ harmonics generate homopolar systems. 
Under non-sinusoidal conditions, no triplen harmonics occur in the line voltages at star connections (because they form homopolar systems) and $U_{l} \leq 3 U_{f}$

Under non-sinusoidal conditions, no triple harmonics occur in the line current at delta connections (because they form homopolar systems) and $I_{l} \leq 3 I_{f}$

\subsection{Voltage harmonics}

According to Faraday's law, the terminal voltage determines the transformer magnetic flux level, as per the relation:

$$
N \frac{d \Phi}{d t}=u(t)
$$

Transferring this equation into the frequency domain it's shows the following relation between the voltage harmonics and the flux components:

$$
\text { (3) } \quad N j(h \omega) \Phi=U_{h}
$$

This equation shows that the flux magnitude is proportional to the voltage harmonic and inversely proportional to the harmonic order $h$.

$P_{n o-l o a d}$ - are the losses due to the core that depend on the load voltage and total harmonic voltage distortion $\left(\mathrm{THD}_{\mathrm{v}}\right)$. If power system's $\mathrm{THD}_{\mathrm{v}}$ is below $5 \%$, and the magnitudes of the voltage harmonics are approx. 2 - 3\% of the fundamental component, the additional no-load loss caused by voltage harmonics is insignificant.

\subsection{Current harmonics}

In most power systems, current harmonics increase losses in the windings and other structural parts of the transformer. - Load losses $\left(P_{\text {load }}\right)$ can be divided into Joule losses, also known as direct current equivalent losses $\left(P_{D C}\right)$ and losses caused by eddy currents in windings, walls or tank, etc. and they depend on the $\mathrm{THD}_{\mathrm{i}}$ current harmonics content. Therefore it results the load losses presented below:

$$
\text { (4) } \quad P_{\text {load }}=P_{D C}+P_{w e}+P_{o s l}
$$

IEEE standard [5] defines the total transformer loss under rated conditions (R-rating) as per the relation:

$$
\text { (5) } \quad P_{\text {load }-R}=I_{R}^{2} R_{D C}+P_{\text {we }-R}+P_{\text {osl }-R}
$$

Transforming total loss in p.u. under normal rated conditions is obtained by relating to the direct current equivalent losses, as per the relation:

$$
\begin{aligned}
& P_{\text {load }-R}=1+\frac{P_{w e-R}}{P_{D C-R}}+\frac{P_{o s l-R}}{P_{D C-R}} \\
& P_{\text {load }-R}=1+P_{w e}(p . u)+P_{o s}(p . u)
\end{aligned}
$$

Presence of harmonics causes an increase of both ohmic and other losses, and these harmonic current losses can be evaluated by using the following formulas:

- Joule losses, also known as direct current equivalent losses:

$$
P_{D C}=3 R_{D C} I_{R}^{2} \sum_{1}^{h \max } \frac{I_{h}^{2}}{I_{R}^{2}}=P_{D C-R} \cdot \sum_{1}^{h \max } \frac{I_{h}^{2}}{I_{R}^{2}}
$$

- winding eddy current losses:

$$
P_{w e}=P_{w e-R} \sum_{1}^{h \max } \frac{I_{h}^{2}}{I_{R}^{2}} h^{2}
$$

current:

Other stray losses caused by harmonic

$$
P_{o s}=P_{o s l-R} \sum_{1}^{h \max } \frac{I_{h}^{2}}{I_{R}^{2}} h^{0.8}
$$

IEEE Standard [8] introduces:

- $\mathrm{F}_{\mathrm{HL}}$ harmonic loss factor defined by the relation:

$$
F_{H L}=\frac{\sum_{1}^{h \max } I_{h}^{2} h^{2}}{\sum_{1}^{h \max } I_{h}^{2}}=\frac{\sum_{1}^{h \max }\left(\frac{I_{h}^{2}}{I_{R}^{2}}\right) h^{2}}{\sum_{1}^{h \max }\left(\frac{I_{h}^{2}}{I_{R}^{2}}\right)}
$$

- $\mathrm{F}_{\mathrm{HL}-\mathrm{STR}}$ harmonic loss factor for other transformer stray losses defined by the relation:

$$
F_{H L-S T R}=\frac{\sum_{1}^{h \max } I_{h}^{2} h^{0.8}}{\sum_{1}^{h \max } I_{h}^{2}}=\frac{\sum_{1}^{h \max }\left(\frac{I_{h}^{2}}{I_{R}^{2}}\right) h^{0.8}}{\sum_{1}^{h \max }\left(\frac{I_{h}^{2}}{I_{R}^{2}}\right)}
$$

Under such circumstances, the transformer total load loss is:

$$
\begin{gathered}
P_{\text {load }-R}=P_{D C-R}+P_{w e-R}+P_{o s l-R} \\
P_{\text {load }-R}=I_{R}^{2} R_{D C}+P_{w e-R}+P_{o s l-R}
\end{gathered}
$$

\subsection{Evaluation of transformer loading capability}

When a transformer is used at non-sinusoidal voltages and currents, its temperature increases and rated power must drop, due to increased losses. Such decrease shall be possible by limiting the transformer total loss under non-sinusoidal current to the amount of sinusoidal voltage and sinusoidal load current losses. In other words, we must determine the transformer maximum admissible harmonic load current, as its loss would be equal to the hot spot loss under sinusoidal current conditions.

A general equation for calculating the transformer power reduction when the transformer carries a harmonic load shall be obtained by enforcing the condition:

$$
P_{\text {load }-R}=P_{\text {load }}
$$


By reporting to the equivalent direct current of the losses it results:

(14) $P_{\text {load }-R}(p . u)=I^{2}\left[1+F_{H L} P_{\text {we }-R}+F_{H L-S T R} P_{o s l-R}\right]$ From this relation we determine the maximum permissible per-unit load current in order to determine the transformer capability decrease with the relation:

$$
I_{\text {der }}=\sqrt{\frac{P_{\text {load }-R}(p . u)}{1+F_{H L} P_{\text {we- } R}+F_{H L-S T R} P_{o s l-R}}}
$$

Simultaneously, the load level must drop until total losses are the same with the sinusoidal losses. The result is the new actual magnitude (expressed in units) the transformer can deliver without exceeding rated losses. The new (reduced) apparent power can be calculated using the relation:

$$
k V A_{\text {derated }}(p . u)=k V A_{\text {rated }}(p \cdot u) \times I_{\text {der }}(p \cdot u)
$$

Therefore, the apparent power percentage reduction shall be:

$$
\text { (17) } \quad \text { Derated }=\left(1-I_{d e r}\right) \times 100 \cdot \%
$$

Underwriter Laboratory (UL) defines the K-factor based on the transformer rated current. The K-factor dependency to rated current is given by the relation:

$$
K_{- \text {factor }}=\sum_{1}^{h \max } \frac{I_{h}^{2}}{I_{1}^{2}} h^{2}=\frac{1}{I_{1}^{2}} \sum_{1}^{h \max } I_{h}^{2} h^{2}
$$

where:

$\mathrm{h}$ is the harmonic order, $\mathrm{I}_{\mathrm{h}}$ is the rms current at harmonic order „h” (ampere), $\mathrm{I}_{1}$ is the rms fundamental current under rated frequency and load conditions (ampere).

The K-factor is dependent on both the magnitude and distribution of the harmonic current. Hence it results that $\mathrm{K}$-factor is a number derived from a numerical calculation based on the summation of the harmonic currents generated by a non-linear load. The network harmonic pollution requires either the transformer decommissioning in order to restore the normal lifetime expectancy or the modernization with a unit designed to accept harmonic currents. The transformer power reduction can be determined based on the $\mathrm{K}$-factor and $\mathrm{THD}_{\mathrm{i}}$ values [10].Knowing the measured K-factor, the harmonic spectrum is defined by:

$$
\text { (19) } I_{1}=\left[\frac{1}{1+T H D^{2}}\right]^{0.5} I_{h}=\frac{\alpha I_{1}}{h}, \alpha=\left[\frac{K-1}{12-0.2145 K}\right]^{0.5}
$$

This calculation is only possible for $\mathrm{k}$-factor $<50$

\subsection{Harmonics effects on transformer heating}

A transformer requested to supply non-sinusoidal loads shall be oversized in order to guarantee that windings temperatures are not exceeded while in service. The top oil temperature rise can be examined using the equation:

$$
\theta_{T O}=\theta_{T O-R} \cdot\left[\frac{P_{D C}+P_{W E-H}}{P_{D C}+P_{W E-50}}\right]^{0.5}
$$

where:

- $\mathrm{P}_{\mathrm{DC}}-\mathrm{DC}$ losses produced by a DC current of the same value that rms fated current;

- $\mathrm{P}_{\mathrm{WE}-50}$ - Winding eddy losses at the rated frequency produced by circulation current (eddy) in the windings;

- $\mathrm{P}_{\mathrm{WE}-\mathrm{H}}-$ Winding eddy losses at load current with harmonics.

The value of this current-caused oversizing factor is calculated by the relation [9]:

$$
K_{w e}=\left[\frac{P_{D C}+P_{W E-H}}{P_{D C}+P_{W E-50}}\right]^{0.5}
$$

By relating the eddy currents losses to the direct current equivalent losses caused by the current actual value we obtain the additional p.u. loss factor:

$$
\text { (22) }(p u)_{W E-50}=\frac{P_{W E-50}}{P_{D C}},(p u)_{W E-H}=\frac{P_{W E-H}}{P_{D C}}
$$

The ratio is:

$$
\begin{gathered}
K_{E F-W E}=\frac{(p u)_{W E-H}}{(p u)_{W E-50}} \text { Or } \\
K_{E F-W E}=\sum_{1}^{h \max } I_{h}^{2}(p u) h^{q}=1+\sum_{2}^{h \max } I_{h}^{2}(p u) h^{q}
\end{gathered}
$$

where $\mathrm{q}$ is exponent for harmonic number.

International standards [11] shows that if the customer can specify rated power and total harmonics distortion $\mathrm{THD}_{\mathrm{i}}$ in p.u. the factor is determined by the relation (23) with:

$$
I_{1}=\left[\frac{1}{1+T H D^{2}}\right]^{0.5}, I_{h}=\frac{\alpha I_{1}}{h}, \quad \alpha=\frac{T H D}{0.463}
$$

\section{CASE STUDY FOR TRANSFORMERS LOADING AND HARMONIC POLLUTION AT SCHELA SUBSTATION}

\subsection{Harmonics values measured}

Power, voltage, current THDi and THDu values measured at the $20 \mathrm{kV}$ Schela substation are shown in the table 3 and the K-factor, current THDi and voltage THDu values measured at the $20 \mathrm{kV}$ Schela substation is shown in the table 4 
THE ANNALS OF "DUNĂREA DE JOS" UNIVERSITY OF GALATI

FASCICLE III, 2020, VOL. 43, NO. 1, ISSN 2344-4738, ISSN-l 1221-454X

Table 3 Values measured in the supply line $20 \mathrm{kV}$

\begin{tabular}{|r|r|r|r|r|r|r|r|}
\hline \multicolumn{1}{|c|}{ Timestamp } & $\begin{array}{c}\text { Apparent } \\
\text { Power } \\
\text { Mean } \\
\text { (kVA) }\end{array}$ & $\begin{array}{c}\text { Current } \\
\text { Avg Mean } \\
\text { (A) }\end{array}$ & $\begin{array}{c}\text { Reactive } \\
\text { Power Mean } \\
\text { (kVAr) }\end{array}$ & $\begin{array}{c}\text { Real Power } \\
\text { Mean (kW) }\end{array}$ & $\begin{array}{c}\text { Voltage L-L } \\
\text { Avg (V) }\end{array}$ & $\begin{array}{c}\text { THD } \\
\text { Current } \\
\text { Mean (\%) }\end{array}$ & $\begin{array}{c}\text { THD } \\
\text { Voltage } \\
\text { Mean (\%) }\end{array}$ \\
\hline $11.09 .20198: 15$ & 90.16 & 3.55 & -76.21 & -39.10 & $20,547.67$ & 101.20 & 1.30 \\
\hline $11.09 .20199: 00$ & 103.87 & 3.84 & -86.69 & -48.94 & $20,638.78$ & 102.07 & 1.25 \\
\hline $11.09 .201910: 00$ & 79.59 & 3.29 & -65.22 & -26.96 & $20,537.62$ & 108.31 & 1.24 \\
\hline $11.09 .201911: 00$ & 111.07 & 4.02 & -75.31 & -78.82 & $20,455.23$ & 84.19 & 1.49 \\
\hline $11.09 .201912: 00$ & 905.49 & 25.66 & -162.09 & -890.86 & $20,499.64$ & 27.04 & 1.53 \\
\hline $11.09 .201913: 00$ & 949.83 & 26.92 & -171.56 & -934.20 & $20,482.00$ & 10.06 & 1.62 \\
\hline $11.09 .201914: 00$ & 935.89 & 26.47 & -167.77 & -920.73 & $20,529.35$ & 9.44 & 1.60 \\
\hline $11.09 .201915: 00$ & 939.56 & 26,60 & -165.61 & -924.83 & $20,500.82$ & 9.51 & 1.56 \\
\hline $11.09 .201916: 00$ & 932.19 & 26.39 & -164.04 & -917.63 & $20,497.91$ & 9.44 & 1.51 \\
\hline $11.09 .201917: 00$ & 951.88 & 26.95 & -174.58 & -935.72 & $20,496.54$ & 9.36 & 1.49 \\
\hline $11.09 .201918: 00$ & 968.13 & 27.33 & -180.43 & -951.15 & $20,559.55$ & 9.61 & 1.39 \\
\hline $11.09 .201919: 00$ & 952.07 & 26.75 & -185.71 & -933.76 & $20,664.53$ & 9.77 & 1.47 \\
\hline $11.09 .201920: 00$ & 995.51 & 28.02 & -188.58 & -977.47 & $20,620.52$ & 9.66 & 1.46 \\
\hline $11.09 .201921: 00$ & $1,003.19$ & 28.28 & -185.72 & -985.84 & $20,590.56$ & 9.42 & 1.90 \\
\hline $11.09 .201922: 00$ & 984.46 & 28.00 & -173.51 & -969.02 & $20,396.29$ & 8.89 & 2.09 \\
\hline $11.09 .201923: 00$ & 986.75 & 28.17 & -166.66 & -972.55 & $20,309.78$ & 8.89 & 1.74 \\
\hline $12.09 .20190: 00$ & 977.68 & 27.85 & -166.95 & -963.30 & $20,363.12$ & 9.08 & 1.68 \\
\hline $12.09 .20191: 00$ & 960.47 & 27.32 & -157.62 & -947.44 & $20,404.14$ & 9.43 & 1.60 \\
\hline $12.09 .20192: 00$ & 980.74 & 27.88 & -167.40 & -966.33 & $20,411.98$ & 9.36 & 1.59 \\
\hline $12.09 .20193: 00$ & 958.50 & 27.26 & -158.04 & -945.37 & $20,397.76$ & 9.38 & 1.56 \\
\hline $12.09 .20194: 00$ & 962.76 & 27.42 & -161.34 & -949.14 & $20,372.63$ & 9.53 & 1.51 \\
\hline $12.09 .20195: 00$ & 955.14 & 27.24 & -151.55 & -943.03 & $20,353.23$ & 9.46 & 1.50 \\
\hline $12.09 .20196: 00$ & 960.61 & 27.36 & -158.61 & -947.39 & $20,370.52$ & 9.37 & 1.48 \\
\hline $12.09 .20197: 00$ & 941.58 & 26.89 & -152.97 & -929.06 & $20,320.70$ & 9.45 & 1.39 \\
\hline $12.09 .20198: 00$ & 947.22 & 26.75 & -162.10 & -933.24 & $20,563.24$ & 9.77 & 1.36 \\
\hline & & & & & & & \\
\hline & & & & & & \\
\hline
\end{tabular}

Table 4 The K-factor, current THDi and voltage THDu values measured

\begin{tabular}{|c|r|r|r|r|r|r|r|r|}
\hline Timestamp & $\begin{array}{c}\text { K-Factor } \\
\text { A Mean } \\
\text { (per unit) }\end{array}$ & $\begin{array}{c}\text { K-Factor } \\
\text { B Mean } \\
\text { (per unit) }\end{array}$ & $\begin{array}{c}\text { K-Factor } \\
\text { C Mean } \\
\text { (per unit) }\end{array}$ & $\begin{array}{c}\text { THD } \\
\text { Current A } \\
\text { Mean (\%) }\end{array}$ & $\begin{array}{c}\text { THD } \\
\text { Current B } \\
\text { Mean (\%) }\end{array}$ & $\begin{array}{c}\text { THD } \\
\text { Current C } \\
\text { Mean (\%) }\end{array}$ & $\begin{array}{c}\text { THD } \\
\text { Voltage } \\
\text { V1 High } \\
\text { (\%) }\end{array}$ & $\begin{array}{c}\text { THD } \\
\text { Voltage } \\
\text { V1 Mean } \\
\text { (\%) }\end{array}$ \\
\hline $11.09 .201913: 00$ & 1.96 & 1.95 & 2.02 & 10.06 & 9.48 & 10.03 & 5.58 & 1.62 \\
\hline $11.09 .201914: 00$ & 1.91 & 1.92 & 1.97 & 9.44 & 8.95 & 9.46 & 1.78 & 1.60 \\
\hline $11.09 .201915: 00$ & 1.91 & 1.91 & 1.96 & 9.51 & 8.99 & 9.38 & 1.76 & 1.56 \\
\hline $11.09 .201916: 00$ & 1.90 & 1.90 & 1.93 & 9.44 & 9.02 & 9.34 & 1.73 & 1.51 \\
\hline $11.09 .201917: 00$ & 1.88 & 1.88 & 1.91 & 9.36 & 8.95 & 9.16 & 1.72 & 1.49 \\
\hline $11.09 .201918: 00$ & 1.88 & 1.88 & 1.91 & 9.61 & 9.16 & 9.40 & 1.59 & 1.39 \\
\hline $11.09 .201919: 00$ & 1.94 & 1.95 & 1.99 & 9.77 & 9.42 & 9.78 & 1.65 & 1.47 \\
\hline $11.09 .201920: 00$ & 1.81 & 1.83 & 1.85 & 9.66 & 9.24 & 9.60 & 1.68 & 1.46 \\
\hline $11.09 .201921: 00$ & 1.73 & 1.73 & 1.80 & 9.42 & 8.89 & 9.46 & 2.12 & 1.90 \\
\hline $11.09 .201922: 00$ & 1.71 & 1.72 & 1.77 & 8.89 & 8.49 & 8.94 & 2.29 & 2.09 \\
\hline $11.09 .201923: 00$ & 1.69 & 1.70 & 1.73 & 8.89 & 8.46 & 8.70 & 1.94 & 1.74 \\
\hline $12.09 .20190: 00$ & 1.70 & 1.73 & 1.75 & 9.08 & 8.82 & 8.85 & 1.86 & 1.68 \\
\hline $12.09 .20191: 00$ & 1.73 & 1.77 & 1.80 & 9.43 & 9.15 & 9.18 & 1.72 & 1.60 \\
\hline $12.09 .20192: 00$ & 1.75 & 1.83 & 1.82 & 9.36 & 9.20 & 9.26 & 1.77 & 1.59 \\
\hline $12.09 .20193: 00$ & 1.76 & 1.81 & 1.82 & 9.38 & 9.19 & 9.25 & 1.73 & 1.56 \\
\hline $12.09 .20194: 00$ & 1.75 & 1.77 & 1.80 & 9.53 & 9.14 & 9.38 & 1.71 & 1.51 \\
\hline $12.09 .20195: 00$ & 1.73 & 1.76 & 1.79 & 9.46 & 9.16 & 9.35 & 1.69 & 1.50 \\
\hline $12.09 .20196: 00$ & 1.74 & 1.79 & 1.81 & 9.37 & 9.15 & 9.37 & 1.62 & 1.48 \\
\hline $12.09 .20197: 00$ & 1.79 & 1.83 & 1.86 & 9.45 & 9.10 & 9.50 & 1.60 & 1.39 \\
\hline $12.09 .20198: 00$ & 1.93 & 1.98 & 2.01 & 9.77 & 9.39 & 9.81 & 1.61 & 1.36 \\
\hline
\end{tabular}


THE ANNALS OF "DUNĂREA DE JOS" UNIVERSITY OF GALATI

FASCICLE III, 2020, VOL. 43, NO. 1, ISSN 2344-4738, ISSN-l 1221-454X

\subsection{Transformer load capability}

The transformer power reduction can be determined based on the $\mathrm{K}$-factor and THDi values measured at the $20 \mathrm{kV}$ Schela substation. Knowing the measured $\mathrm{K}$-factor, the harmonic spectrum is defined by (19) and the apparent power percentage reduction by (16) and the results is shown in table 5 . The de-rating of the apparent power percentage is between $24-32 \%$, the high values being for a large factor $\mathrm{k}$. In practice, the transformer would need to be de-rated to 76 - $68 \%$ of nominal power rating when supplying a non-linear load.

Table 5 Derating transformer

\begin{tabular}{|c|c|c|c|c|c|c|c|c|c|c|c|c|c|c|c|}
\hline Timestamp & $\begin{array}{c}\text { K- } \\
\text { (per } \\
\text { unit) }\end{array}$ & $\begin{array}{c}\text { THD } \\
\text { i } \\
(\%)\end{array}$ & $\alpha=\left[\frac{K-}{12-0.2]}\right.$ & $\mathrm{I}_{1}$ & $\mathrm{I}_{3}$ & $\mathrm{I}_{5}$ & $\mathrm{I}_{7}$ & $\mathrm{I}_{9}$ & $\mathrm{I}_{11}$ & $\mathrm{I}_{13}$ & $\mathrm{I}_{15}$ & $\mathrm{I}_{17}$ & $\mathrm{I}^{2}$ & I & 葋 \\
\hline $\begin{array}{r}11.09 .2019 \\
13: 00: 00\end{array}$ & 1.96 & 10.06 & 0.29 & 0.99 & 0.10 & 0.06 & 0.04 & 0.03 & 0.03 & 0.02 & 0.02 & 0.02 & 1.73 & 1.32 & 31.65 \\
\hline $\begin{array}{r}11.09 .2019 \\
14: 00: 00\end{array}$ & 1.91 & 9.44 & 0.28 & 1.00 & 0.09 & 0.06 & 0.04 & 0.03 & 0.03 & 0.02 & 0.02 & 0.02 & 1.70 & 1.30 & 30.22 \\
\hline $\begin{array}{r}11.09 .2019 \\
15: 00: 00 \\
\end{array}$ & 1.91 & 9.51 & 0.28 & 1.00 & 0.09 & 0.06 & 0.04 & 0.03 & 0.03 & 0.02 & 0.02 & 0.02 & 1.69 & 1.30 & 30.17 \\
\hline $\begin{array}{r}11.09 .2019 \\
16: 00: 00\end{array}$ & 1.90 & 9.44 & 0.28 & 1.00 & 0.09 & 0.06 & 0.04 & 0.03 & 0.03 & 0.02 & 0.02 & 0.02 & 1.69 & 1.30 & 29.89 \\
\hline $\begin{array}{r}11.09 .2019 \\
17: 00: 00\end{array}$ & 1.88 & 9.36 & 0.28 & 1.00 & 0.09 & 0.05 & 0.04 & 0.03 & 0.02 & 0.02 & 0.02 & 0.02 & 1.67 & 1.29 & 29.27 \\
\hline $\begin{array}{r}11.09 .2019 \\
18: 00: 00 \\
\end{array}$ & 1.88 & 9.61 & 0.28 & 1.00 & 0.09 & 0.05 & 0.04 & 0.03 & 0.02 & 0.02 & 0.02 & 0.02 & 1.67 & 1.29 & 29.35 \\
\hline $\begin{array}{r}11.09 .2019 \\
19: 00: 00\end{array}$ & 1.94 & 9.77 & 0.29 & 1.00 & 0.09 & 0.06 & 0.04 & 0.03 & 0.03 & 0.02 & 0.02 & 0.02 & 1.72 & 1.31 & 31.15 \\
\hline $\begin{array}{r}11.09 .2019 \\
20: 00: 00\end{array}$ & 1.81 & 9.66 & 0.26 & 1.00 & 0.09 & 0.05 & 0.04 & 0.03 & 0.02 & 0.02 & 0.02 & 0.02 & 1.62 & 1.27 & 27.10 \\
\hline $\begin{array}{r}11.09 .2019 \\
21: 00: 00\end{array}$ & 1.73 & 9.42 & 0.25 & 1.00 & 0.08 & 0.05 & 0.04 & 0.03 & 0.02 & 0.02 & 0.02 & 0.01 & 1.56 & 1.25 & 24.86 \\
\hline $\begin{array}{r}11.09 .2019 \\
22: 00: 00\end{array}$ & 1.71 & 8.89 & 0.25 & 1.00 & 0.08 & 0.05 & 0.04 & 0.03 & 0.02 & 0.02 & 0.02 & 0.01 & 1.54 & 1.24 & 24.23 \\
\hline $\begin{array}{r}11.09 .2019 \\
23: 00: 00\end{array}$ & 1.69 & 8.89 & 0.24 & 1.00 & 0.08 & 0.05 & 0.03 & 0.03 & 0.02 & 0.02 & 0.02 & 0.01 & 1.53 & 1.24 & 23.53 \\
\hline $\begin{array}{r}12.09 .2019 \\
0: 00: 00 \\
\end{array}$ & 1.70 & 9.08 & 0.25 & 1.00 & 0.08 & 0.05 & 0.03 & 0.03 & 0.02 & 0.02 & 0.02 & 0.01 & 1.53 & 1.24 & 23.88 \\
\hline $\begin{array}{r}12.09 .2019 \\
1: 00: 00\end{array}$ & 1.73 & 9.43 & 0.25 & 1.00 & 0.08 & 0.05 & 0.04 & 0.03 & 0.02 & 0.02 & 0.02 & 0.01 & 1.56 & 1.25 & 24.80 \\
\hline $\begin{array}{r}12.09 .2019 \\
2: 00: 00\end{array}$ & 1.75 & 9.36 & 0.25 & 1.00 & 0.08 & 0.05 & 0.04 & 0.03 & 0.02 & 0.02 & 0.02 & 0.01 & 1.57 & 1.25 & 25.49 \\
\hline $\begin{array}{r}12.09 .2019 \\
3: 00: 00 \\
\end{array}$ & 1.76 & 9.38 & 0.26 & 1.00 & 0.08 & 0.05 & 0.04 & 0.03 & 0.02 & 0.02 & 0.02 & 0.01 & 1.58 & 1.26 & 25.70 \\
\hline $\begin{array}{r}12.09 .2019 \\
4: 00: 00 \\
\end{array}$ & 1.75 & 9.53 & 0.25 & 1.00 & 0.08 & 0.05 & 0.04 & 0.03 & 0.02 & 0.02 & 0.02 & 0.01 & 1.57 & 1.25 & 25.19 \\
\hline $\begin{array}{r}12.09 .2019 \\
5: 00: 00\end{array}$ & 1.73 & 9.46 & 0.25 & 1.00 & 0.08 & 0.05 & 0.04 & 0.03 & 0.02 & 0.02 & 0.02 & 0.01 & 1.55 & 1.25 & 24.61 \\
\hline $\begin{array}{r}12.09 .2019 \\
6: 00: 00 \\
\end{array}$ & 1.74 & 9.37 & 0.25 & 1.00 & 0.08 & 0.05 & 0.04 & 0.03 & 0.02 & 0.02 & 0.02 & 0.01 & 1.57 & 1.25 & 25.18 \\
\hline $\begin{array}{r}12.09 .2019 \\
7: 00: 00\end{array}$ & 1.79 & 9.45 & 0.26 & 1.00 & 0.09 & 0.05 & 0.04 & 0.03 & 0.02 & 0.02 & 0.02 & 0.02 & 1.60 & 1.26 & 26.44 \\
\hline $\begin{array}{r}12.09 .2019 \\
8: 00: 00\end{array}$ & 1.93 & 9.77 & 0.28 & 1.00 & 0.09 & 0.06 & 0.04 & 0.03 & 0.03 & 0.02 & 0.02 & 0.02 & 1.71 & 1.31 & 30.93 \\
\hline
\end{tabular}

\subsection{Evaluation of harmonic-polluted transformer thermal overload}

Evaluation of harmonic-polluted transformer thermal overload is based by equations (20), (21) and (24) and make with the relation:

where was determine harmonics up to 17 th order and the result is shown in table 6 .

$$
\frac{\theta_{T O}-\theta_{T O-R}}{\theta_{T O-R}} 100=\left\{\left[\frac{P_{D C}+P_{W E-H}}{P_{D C}+P_{W E-50}}\right]^{0.5}-1\right\} 100 \%
$$


THE ANNALS OF "DUNĂREA DE JOS" UNIVERSITY OF GALATI

FASCICLE III, 2020, VOL. 43, NO. 1, ISSN 2344-4738, ISSN-l 1221-454X

Table 6 Calculation of harmonics and overheating of the transformer

\begin{tabular}{|c|c|c|c|c|c|c|c|c|c|c|c|c|c|c|}
\hline Timestamp & $\begin{array}{c}\text { THD } \\
\text { Current A } \\
\text { Mean } \\
(\%)\end{array}$ & $\begin{array}{l}\text { THD } \\
\text { Volta } \\
\text { ge V1 } \\
\text { Mean } \\
(\%)\end{array}$ & $\alpha=\frac{T H D}{0.463}$ & $\mathrm{I}_{1}$ & $\mathrm{I}_{3}$ & $\mathrm{I}_{5}$ & $\mathrm{I}_{7}$ & $\mathrm{I}_{9}$ & $\mathrm{I}_{11}$ & $\mathrm{I}_{13}$ & $\mathrm{I}_{15}$ & $\mathrm{I}_{17}$ & EIrime & $\frac{615:-6 h s-1}{100 \%-\pi}$ \\
\hline 11.09.2019 8:00:00 & 101.20 & 1.30 & 2.19 & 0.70 & 0.51 & 0.31 & 0.22 & 0.17 & 0.14 & 0.12 & 0.10 & 0.09 & 7.89 & 180.89 \\
\hline 11.09.20199:00:00 & 102.07 & 1.25 & 2.20 & 0.70 & 0.51 & 0.31 & 0.22 & 0.17 & 0.14 & 0.12 & 0.10 & 0.09 & 7.95 & 181.90 \\
\hline 11.09.2019 10:00:00 & 108.31 & 1.24 & 2.34 & 0.68 & 0.53 & 0.32 & 0.23 & 0.18 & 0.14 & 0.12 & 0.11 & 0.09 & 8.35 & 188.89 \\
\hline 11.09.2019 11:00:00 & 84.19 & 1.49 & 1.82 & 0.76 & 0.46 & 0.28 & 0.20 & 0.15 & 0.13 & 0.11 & 0.09 & 0.08 & 6.66 & 158.00 \\
\hline 11.09.2019 12:00:00 & 27.04 & 1.53 & 0.58 & 0.97 & 0.19 & 0.11 & 0.08 & 0.06 & 0.05 & 0.04 & 0.04 & 0.03 & 1.93 & 39.04 \\
\hline 11.09.2019 13:00:00 & 10.06 & 1.62 & 0.22 & 0.99 & 0.07 & 0.04 & 0.03 & 0.02 & 0.02 & 0.02 & 0.01 & 0.01 & 1.14 & 6.64 \\
\hline 11.09.2019 14:00:00 & 9.44 & 1.60 & 0.2 & 1.00 & 0.07 & 0.04 & 0.03 & 0.02 & 0.02 & 0.02 & 0.01 & 0.01 & 1.12 & 5.88 \\
\hline 11.09.2019 15:00:00 & 9.51 & 1.56 & 0.21 & 1.00 & 0.07 & 0.04 & 0.03 & 0.02 & 0.02 & 0.02 & 0.01 & 0.01 & 1.12 & 5.96 \\
\hline 11.09.2019 16:00:00 & 9.44 & 1.51 & 0.20 & 1.00 & 0.07 & 0.04 & 0.03 & 0.02 & 0.02 & 0.02 & 0.01 & 0.01 & 1.12 & 5.88 \\
\hline 11.09.2019 17:00:00 & 9.36 & 1.49 & 0.20 & 1.00 & 0.07 & 0.04 & 0.03 & 0.02 & 0.02 & 0.02 & 0.01 & 0.01 & 1.12 & 5.78 \\
\hline 11.09.2019 18:00:00 & 9.61 & 1.39 & 0.21 & 1.00 & 0.07 & 0.04 & 0.03 & 0.02 & 0.02 & 0.02 & 0.01 & 0.01 & 1.13 & 6.09 \\
\hline 11.09.2019 19:00:00 & 9.77 & 1.47 & 0.21 & 1.00 & 0.07 & 0.04 & 0.03 & 0.02 & 0.02 & 0.02 & 0.01 & 0.01 & 1.13 & 6.28 \\
\hline $11.09 .201920: 00: 00$ & 9.66 & 1.46 & 0.21 & 1.00 & 0.07 & 0.04 & 0.03 & 0.02 & 0.02 & 0.02 & 0.01 & 0.01 & 1.13 & 6.14 \\
\hline 11.09.2019 21:00:00 & 9.42 & 1.90 & 0.20 & 1.00 & 0.07 & 0.04 & 0.03 & 0.02 & 0.02 & 0.02 & 0.01 & 0.01 & 1.12 & 5.85 \\
\hline 11.09.2019 22:00:00 & 8.89 & 2.09 & 0.19 & 1.00 & 0.06 & 0.04 & 0.03 & 0.02 & 0.02 & 0.01 & 0.01 & 0.01 & 1.11 & 5.24 \\
\hline 11.09.2019 23:00:00 & 8.89 & 1.74 & 0.19 & 1.00 & 0.06 & 0.04 & 0.03 & 0.02 & 0.02 & 0.01 & 0.01 & 0.01 & 1.11 & 5.24 \\
\hline $12.09 .20190: 00: 00$ & 9.08 & 1.68 & 0.20 & 1.00 & 0.07 & 0.04 & 0.03 & 0.02 & 0.02 & 0.02 & 0.01 & 0.01 & 1.11 & 5.46 \\
\hline $12.09 .20191: 00: 00$ & 9.43 & 1.60 & 0.20 & 1.00 & 0.07 & 0.04 & 0.03 & 0.02 & 0.02 & 0.02 & 0.01 & 0.01 & 1.12 & 5.87 \\
\hline 12.09.2019 2:00:00 & 9.36 & 1.59 & 0.20 & 1.00 & 0.07 & 0.04 & 0.03 & 0.02 & 0.02 & 0.02 & 0.01 & 0.01 & 1.12 & 5.79 \\
\hline $12.09 .20193: 00: 00$ & 9.38 & 1.56 & 0.20 & 1.00 & 0.07 & 0.04 & 0.03 & 0.02 & 0.02 & 0.02 & 0.01 & 0.01 & 1.12 & 5.81 \\
\hline $12.09 .20194: 00: 00$ & 9.53 & 1.51 & 0.21 & 1.00 & 0.07 & 0.04 & 0.03 & 0.02 & 0.02 & 0.02 & 0.01 & 0.01 & 1.12 & 5.99 \\
\hline 12.09.2019 5:00:00 & 9.46 & 1.50 & 0.20 & 1.00 & 0.07 & 0.04 & 0.03 & 0.02 & 0.02 & 0.02 & 0.01 & 0.01 & 1.12 & 5.90 \\
\hline $12.09 .20196: 00: 00$ & 9.37 & 1.48 & 0.20 & 1.00 & 0.07 & 0.04 & 0.03 & 0.02 & 0.02 & 0.02 & 0.01 & 0.01 & 1.12 & 5.80 \\
\hline $12.09 .20197: 00: 00$ & 9.45 & 1.39 & 0.20 & 1.00 & 0.07 & 0.04 & 0.03 & 0.02 & 0.02 & 0.02 & 0.01 & 0.01 & 1.12 & 5.89 \\
\hline $12.09 .20198: 00: 00$ & 9.77 & 1.36 & 0.21 & 1.00 & 0.07 & 0.04 & 0.03 & 0.02 & 0.02 & 0.02 & 0.01 & 0.01 & 1.13 & 6.28 \\
\hline
\end{tabular}

Table 7 The energy loss calculation

\begin{tabular}{|c|c|c|c|c|c|c|c|c|c|c|c|c|c|c|}
\hline p & 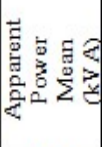 & 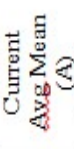 & 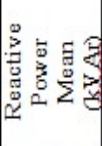 & 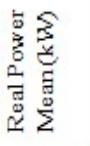 & 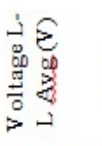 & 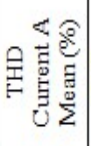 & 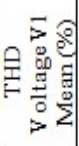 & 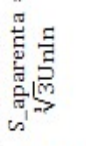 & 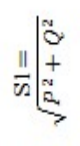 & $\underbrace{\left[\begin{array}{l}w \\
1 \\
\tilde{w}\end{array}\right.}_{\| 11}$ & & & & \\
\hline $11.09 .20198: 150$ & 90.16 & 3.55 & -76.21 & 9.10 & 547.6 & 1.20 & 1.30 & 126.44 & 85.65 & 3.01 & 0.46 & 0.31 & 2.78 & 0.49 \\
\hline & 103.87 & 3.84 & -86.69 & & $20,638.7$ & 2.07 & 1.25 & 137 & 99.55 & 4.37 & 0.49 & 0.36 & 94.15 & 0.45 \\
\hline & .59 & 3.29 & -65.22 & 6 & 537.6 & 108.31 & 1.24 & 11 & 70.57 & 3.18 & 0.38 & 23 & 92.98 & 0.58 \\
\hline & 111.07 & 4.02 & 5.31 & 8.82 & $0,455.2$ & 84.19 & 1.49 & 142.49 & 09.01 & 91.76 & 12 & .55 & 91.44 & 0 \\
\hline & 5.49 & 66 & 2.09 & 90.8 & & .04 & 1.53 & & 05.49 & 100.52 & 0.98 & 0.98 & 97.08 & 0 \\
\hline$: 00$ & 949.83 & 26.92 & -171.56 & -934.2 & $20,482.0$ & 10.06 & 1.62 & 954.97 & 949.82 & 99.05 & 0.98 & 0.98 & 74.57 & 0.00 \\
\hline & 935.89 & 26.47 & -167.77 & -920.7 & $20,529.3$ & 9.44 & 1.60 & 941.09 & 935.89 & 98.79 & 0.98 & 0.98 & 71.07 & 0.00 \\
\hline & 9.56 & 26.60 & -165.61 & & 205 & 9.51 & 1.56 & 944.37 & 939.54 & 95.30 & 0.98 & 0.98 & 68.96 & 0.00 \\
\hline & 2.19 & 26 & -1 & & & 9.44 & 1.51 & 93 & 2.17 & 5.61 & 0.98 & 0.98 & 68.81 & 0.00 \\
\hline & 1.88 & 26.95 & -174.58 & 7 & $20,496.5$ & 9.36 & 1.49 & 956.73 & 51.87 & 96.31 & 0.98 & 0.98 & 68.81 & 0.00 \\
\hline & 8.13 & 27.33 & -180.43 & & & 9.61 & 1.39 & 973.39 & 58.12 & 101.24 & 0.98 & 0.98 & 73.85 & 0.00 \\
\hline 9:00 & 952.07 & 26.75 & -185.71 & -933.7 & $20,664.5$ & 9.77 & 1.47 & 957.43 & 952.05 & 101.34 & 0.98 & 0.98 & 74.78 & 0.00 \\
\hline & 95.51 & 28.02 & -188.58 & -977.4 & 20,6 & 9.66 & 1.46 & 1000.5 & 995.50 & 100.87 & 0.98 & 0.98 & 73.84 & 0.00 \\
\hline & $1,003.1$ & 28.28 & -185.72 & & 20, & 9.42 & 1.90 & 1000.4 & 1003.1 & 103.26 & 0.98 & 0.98 & 74.15 & 0.00 \\
\hline & 4.46 & 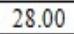 & 73.51 & & & 8.89 & 2.09 & \begin{tabular}{|l|}
989.01 \\
\end{tabular} & 43 & 95.07 & 0.98 & 0.98 & 65.02 & 0.00 \\
\hline 00 & 6.75 & 28.17 & -166.66 & 5 & $20,309.7$ & 8.89 & 1.74 & 991.02 & 86.73 & 92.14 & 0.99 & 0.98 & 63.01 & 0.00 \\
\hline & 7.68 & 28.85 & -166.95 & 63.3 & 20,3 & 9.08 & 1.68 & 982.25 & 77.66 & 94.87 & 0.99 & 0.98 & 66.13 & 0.00 \\
\hline $1: 00$ & 960.47 & 27.32 & -157.62 & -963.3 & $20,404.1$ & 9.43 & 1.60 & 965.49 & 960.47 & 98.37 & 0.99 & 0.98 & 70.73 & 0.00 \\
\hline & 30.74 & 27.88 & -167.40 & -966.3 & & 9.36 & 1.59 & 985.61 & 980.72 & 98.03 & 0.99 & 0.98 & 70.08 & 0.00 \\
\hline & 8.50 & 27.26 & -158.04 & & & 9.38 & 1.56 & \begin{tabular}{|l|l}
963.08 \\
\end{tabular} & 958.49 & 93.99 & 0.99 & 0.98 & 67.31 & 0.00 \\
\hline & .76 & 27. & & & & 9.53 & 1.51 & .66 & 962.75 & 97.33 & 0.99 & 0.98 & 70.55 & 0.00 \\
\hline & 5.14 & 27.24 & -151.55 & & & 9.46 & 1.50 & 960.27 & 955.13 & 99.18 & 0.99 & .98 & 71.46 & 0.00 \\
\hline & 0.61 & 27.36 & -158.61 & 3 & & 9.37 & 1.48 & 965.50 & 60.57 & 97.40 & 0.99 & 98 & 69.68 & 0.00 \\
\hline$: 00$ & 941.58 & 26.89 & -152.97 & -929.0 & $20,320.7$ & 9.45 & 1.39 & \begin{tabular}{|l|l}
946.49 \\
\end{tabular} & 941.57 & 96.39 & 0.99 & 0.98 & 69.40 & 0.00 \\
\hline $12.09 .20198: 00$ & 947.22 & 26.75 & -162.10 & -933.2 & $20,563.2$ & 9.77 & 1.36 & 952.67 & 947.21 & 101.82 & 0.99 & 0.98 & 75.16 & 0.00 \\
\hline \multicolumn{2}{|l|}{ Ulat } & & -36733 & -19102 & & & & 19830 & 19572 & 2332. & & & 1806 & 1.72 \\
\hline
\end{tabular}




\subsection{Analysis of powers and tariffs thereof}

Due to harmonics content under non-sinusoidal conditions, the apparent power is defined by the voltage and current rms values as per the equation below:

(26) $S^{2}=\sum U_{n}^{2} \sum I_{n}^{2}>\left(\sum U_{n} I_{n} \cos \varphi_{n}\right)^{2}+\left(\sum U_{n} I_{n} \sin \varphi_{n}\right)^{2}$ C. Budeanu introduces the concept of deformed or distortion which consists mainly in the cross-product of voltage and current harmonics of different orders and it will be reduced to zero if the harmonics are reduced to zero, that is under sinusoidal conditions. The power parallelogram is shown in the figure 3 and equation is:

$$
D=\sqrt{S^{2}-\left(P^{2}+Q^{2}\right)}
$$

In consideration of energy, the national authority publishes the tariffs for power distribution services provided by the main distribution operators and also the reactive power price [12]. Tariffs of reactive power for industrial users in Romania are calculated based on Budeanu's reactive power definition:

$$
Q=\sum_{1}^{N} U_{h} I_{h} \sin \varphi_{h}
$$

Where: Uh, Ih and $\sin \varphi_{\mathrm{h}}$ are values for the $\mathrm{h}$-th harmonics (considered up to $\mathrm{N}$-th order harmonic)

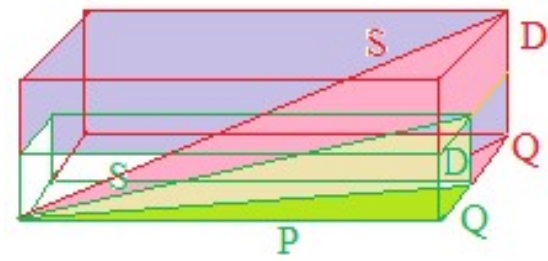

Fig.3 The power parallelogram. Regulated areas by standards (green) and the punishable area (red) in the powers parallelogram

The actual average power factor, corresponding strictly to the reactive power consumption, considered in invoicing determined on active power $\mathrm{Wa}$ and reactive power $\mathrm{Wr}$ established for the invoicing period is

$$
\lambda^{a}=\frac{W_{a}}{\sqrt{W_{a}^{2}+W_{r}^{2}}}
$$

As a matter of fact, when referring the neutral power factor $\lambda^{a}>0.9$ as long as a current harmonic distortion exists, there shall occur power losses due to deformed power, if such deformed power is over the THD rated value. Nowadays, reactive power tariff in Romania is based on the assumption that the transfer of one $\mathrm{kVAr}$ through electric networks causes a constant active power loss of $0.1 \mathrm{~kW}$, irrespective of the consumer's voltage connection.
For a real consumer, with an unbalanced and nonlinear load, the above-defined power factor results in a lower invoice value (the power factor $\lambda^{a}$ does not include the deformed power and only considers the active and reactive power). Moreover, it does not provide accurate information on the required investment for further decrease of invoices by taking appropriate actions to locally produce reactive power and to filter harmonics. Additional losses caused by the harmonics flow in the network are paid for by all consumers, proportionally to the power consumption, irrespective of the actions taken by the users for filtering the harmonics generated by their operation. The currently used numerical meters (especially those designed in America) include power factor definitions based on the apparent power referring to complementary (non-active) power, to which additional network power losses actually belong and which should be mitigated as per the relation:

$$
\text { (30) } \lambda=\frac{\sqrt{S^{2}-P^{2}}}{S}
$$

The link between the two factors being:

$$
\lambda^{a}=\sqrt{1-\lambda^{2}}
$$

Due to a weak power factor, the transmission capability is reduced and a power loss is contemplated. Especially under the current nonlinear load environment, the power factor is that takes care of the reactive power demand, due to the fundamental waveform, and also of the distorted waveform. The displacement power factor is the power factor due to the phase shift between voltage and current at the fundamental line frequency. An image of the regulated area (green) and the punishable area (red) in the powers parallelogram is shown in the figure 3 . In case of harmonics occurrence over the regulated limits, the relating costs should be split between the dealer and the consumer.

A simple and largely used way of splitting liabilities and costs of the issues relating to power quality, including reactive power, is to claim that voltage is the power supplier's responsibility, while current is the consumer's responsibility.

The cost of losses is approximately proportionate to the square of currents. The power loss due to THDi can be assessed by the relation:

Lost power $=[1-$ [regulated THDi2 / operating THDi2] $x$ total consumption of deformed power.

Power loss due to a power factor which is below the regulated power factor can be assessed by the relation:

Lost power $=[1-$ [operating $P F /$ regulated $P F] x$ total reactive power consumption. 
Out of data analysis it results the existence of a power loss due especially to THDi shown in table 7 . The energy loss calculation was performed in table 7 only for the THDi values outside the standardized limits shown in table 1 and with a power factor of less than 0.9 .

\section{CONCLUSIONS}

The result of the analysis of the measured values and the calculations performed regarding the reduction of the power of the transformer, the increase of the temperature of the transformer oil and the circulation of powers, the following conclusions can be drawn: 1. Regarding the transformer load capacity and the power reduction determined based on the K-factor and THDi values measured at the $20 \mathrm{kV}$ Schela station, it results in a de-rating of the apparent power percentage between $24-32 \%$;

2. The evaluation of the thermal overload transformer based on knowledge of the total harmonic distortion THDi p.u. shows that the thermal overload of the transformer is around $6 \%$ for THDi values between $9-10 \%$ and $180-190 \%$ for THDi values over $100 \%$;

3. Concerning the power circulation, the presence of the harmonic current leads to high values of the deforming power between $60-70 \mathrm{kVAD}$ at an average active power of $950 \mathrm{~kW}$ and a power factor greater than 0.9 and at values over $90 \mathrm{kVAD}$ at a reduced power factor;

4. The proposed calculation relationship for energy loss assessment due to non-compliance of THDi within the limits imposed by standards indicates that at an active energy of $19102 \mathrm{kWh}$ there is a lost energy of $1806 \mathrm{kVADh}$ due to the deforming power;

5. The proposed calculation relationship for energy loss assessment due to non-compliance of power factor within the limits imposed by standards indicates that at an active energy of $19102 \mathrm{kWh}$ there is a lost energy only of $1.72 \mathrm{kVARh}$ due to the reactive power.

The general conclusion is that measures must be taken to limit the harmonic current by the responsible factors and / or to penalize the consumer if they do not fall within the standardized limits.

\section{REFERENCES}

IEEE 1100-1992. Recommended Practice for Powering and Grounding Sensitive Electronic Equipment.

Ali Zeraatparvar; Taghi Sami; F. Abbasi Raheli Evaluation of performance of distribution transformers supplying non-linear loads currents: Effect of oversizing- 2011 10th International Conference on Environment and Electrical Engineering - $\quad$ Rome, ItalyDOI:10.1109/EEEIC.2011.5874586 Publisher: IEEE.
K. D. Patil; W. Z. Gandhare-Effects of harmonics in distribution systems on temperature rise and life of XLPE power cables-2011 International Conference on Power and Energy Systems Chennai, India DOI: 10.1109/ICPES.2011.6156680 Publisher: IEEE.

Zhemin Lin; Xuchang Zhang; Xun Mao; Yong Zhan; Xiaotao Peng; Bin Zhang-Economic evaluation of harmonic cost - 2016 IEEE 8th International Power Electronics and Motion Control Conference (IPEMC-ECCE Asia)-Hefei, China - DOI: 10.1109/IPEMC.2016.7512365 Publisher: IEEE.

Dejan Pejovski, Krste Najdenkoski, Mihail Digalovski - Impact of different harmonic loads on distribution transformers - Procedia Engineering 202 (2017) 76-87.

Jaspreet Singh Sanjeev Singh Amanpreet Singh Effect of Harmonics on Distribution Transformer Losses and Capacity - International Journal of Engineering Technology Science and Research IJETSR www.ijetsr.com ISSN 2394 3386 Volume 4, Issue 6, June 2017.

IEEE standard 512-1992, IEEE recommended Practices and requirements for Harmonic control in Electrical Power System

PAN Chao, KONG Lingguo, LI Zhenxin, ZHENG Qing, WANG Zezhong - Analysis Based on Improved Method for Transformer Harmonic Losses - Energy Pricedia 16 (2012) 1845 - 1851.

IEEE standard C57.110 - 1998, "IEEE recommended Practice for Establishing Transformer Capability when Supplying Non Sinusoidal Currents.

LES100070-ZB - Loading transformers with nonsinusoidal currents - 2007 ABB https://library.e.abb.com/public/be0cadaf6a6708 fcc1257792005162cb/Loading_transformers_wit h_non_sinusoidal_currents_KFactor.pdf.

IEC 61000-2-4:2002 Electromagnetic compatibility (EMC) - Part 2-4: Environment - Compatibility levels in industrial plants for low-frequency conducted disturbances.

MO-152/26.02.2019

https://www.anre.ro/ro/legislatie/legislatie/pretur i-si-tarife-ee/energie-electrica-2019. 